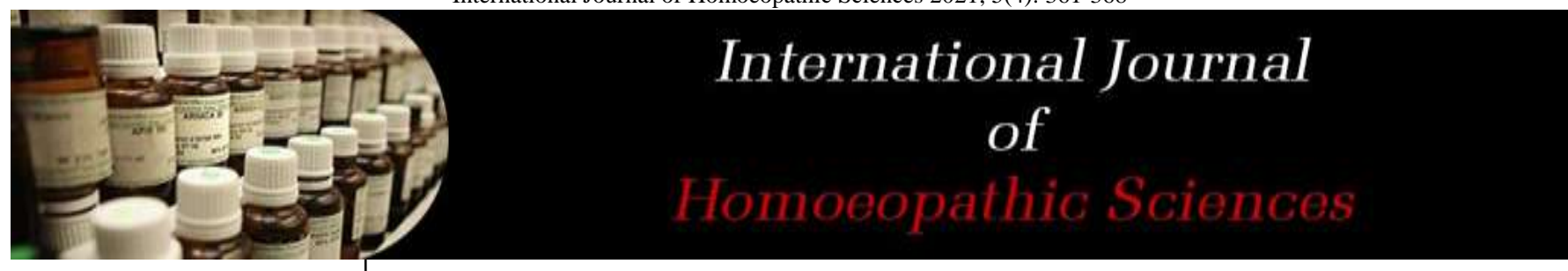

E-ISSN: 2616-4493 P-ISSN: 2616-4485 www.homoeopathicjournal.com IJHS 2021; 5(4): 361-368 Received: 08-07-2021 Accepted: 03-09-2021

Dr. Rajani Kumari PGT. Department Practice of Medicine, R.B.T.S Govt. Homoeopathic Medical College and Hospital, Muzaffarpur, Bihar, India
Corresponding Author: Dr. Rajani Kumari PGT. Department Practice of Medicine, R.B.T.S Govt. Homoeopathic Medical College and Hospital, Muzaffarpur, Bihar, India

\section{Migraine a modern problem and its homoeopathic approach}

\section{Dr. Rajani Kumari}

DOI: https://doi.org/10.33545/26164485.2021.v5.i4f.495

\begin{abstract}
In this modern era, conventional and bio-chemical intervention are not having good result in cases of migraine. Homoeopathy and other complementary medicine are having a good result in cases of Migraine and different kind of headaches. As Homoeopathy treats the person as a whole and depends upon the individualization, we get better result in homoeopathy other than other system of medicine. The main aim of the study to assess the effect of Homoeopathy medicine in cases of Migraine by using the Materia medica and repertory.
\end{abstract}

Keywords: Migraine, headaches, management, homoeopathy

\section{Introduction}

Headache is among the most common reasons that patients seek medical attention. Migraine accounts for the $16 \%$ of the primary headaches as per the classification system developed by the International Headache Society, who characterized headache as primary or secondary. Primary headaches are those in which headache and its associated features are the disorder in itself and often results in considerable disability and a decrease in the patient's quality of life. Migraine is a benign and recurring syndrome of headache associated with other symptoms of neurologic dysfunction in varying admixtures.

\section{Epidemiology}

Migraine is the second most common cause of headache, afflicting approximately $15 \%$ of women and $6 \%$ of men. The American Migraine Foundation estimates that more than 36 million Americans get them, women 3 times more often than men do. Most people start having migraine headaches between ages 10 and 40. However, many women find that their migraines improve or disappear after age 50. They generally last between 4 and 72 hours. It is usually an episodic headache that is associated with certain features such as sensitivity to light, sound, or movement; nausea and vomiting often accompany the headache.

\section{Pathophysiology}

The pathophysiology of migraine probably relates to the neurotransmitter serotonin. Headache may result from release of neuropeptides acting as neurotransmitters at trigeminal nerve branches, leading to an inflammatory process; another possible mechanism involves activation of the dorsal raphe nucleus. Imaging studies have revealed changes in brainstem regions involved in sensory modulation, suggesting that migraine relates to a failure of normal sensory processing. The brain of the migraineur is particularly sensitive to environmental and sensory stimuli; migraine-prone patients do not habituate easily to sensory stimuli. This sensitivity is amplified in females during the menstrual cycle. Headache can be initiated or amplified by various triggers, including glare, bright lights, sounds, or other afferent stimulation; hunger; excess stress; physical exertion; stormy weather or barometric pressure changes; hormonal fluctuations during menses; lack of or excess sleep; and alcohol or other chemical stimulation. Knowledge of a patient's susceptibility to specific triggers can be useful in management strategies involving lifestyle adjustments.

\section{What Causes Migraine Headache?}

Migraine headaches are a symptom of an overall condition known as migraine. The exact cause of migraine headaches, although they seem to be related to changes in 
the brain as well as to genes that run in families. One can even inherit the triggers that give migraine headaches, like fatigue, bright lights, weather changes, and others. For many years, scientists believed migraines resulted from changes in blood flow in the brain. Many now think that they happen because of flaws in the brain passed down from your parents. A migraine starts when overactive nerve cells send out signals that activate the trigeminal nerve, the nerve that supplies sensation to your head and face. Activation of the nerve causes release of certain chemicals like serotonin and calcitonin gene-related peptide (CGRP). CGRP causes blood vessels in the lining of the brain to swell. This releases neurotransmitters that create inflammation and pain.

\section{Risk Factors}

Some common migraine triggers include:

- Sex. Women are three times more likely to have migraines.

- Family history. If you have a family member with migraines, then you have a good chance of developing them too.

- Age. Migraines can begin at any age, though the first often occurs during adolescence. Migraines tend to peak during your 30 s, and gradually become less severe and less frequent in the following decades.

- Stress and anxiety: Stress, depression, anxiety, excitement, and shock can trigger a migraine. When stressed, our brain releases chemicals that can cause the blood vessel changes that can lead to a migraine.

- Foods: Some foods and drinks, such as aged cheese, alcohol. Processed, fermented, pickled, or marinated foods, Peanuts and other nuts and seed, Onions, Meats containing nitrates (bacon, hot dogs, salami, cured meats), Foods with tyramine, which includes red wine, smoked fish, chicken livers, figs, and certain beans, Chocolates and Fruits (avocado, banana, citrus fruit)

- Caffeine: Getting too much or withdrawing from it can cause headaches when the level in your body abruptly drops. Blood vessels seem to get used to caffeine, and when you do not have any, you may get a headache. Caffeine itself can be a treatment for acute migraine attacks.

- Changes in weather: Storm fronts, changes in barometric pressure, strong winds, or changes in altitude can all trigger a migraine. Flickering screens, strong smells, second-hand smoke, and loud noises can set off a migraine. Stuffy rooms, temperature changes, and bright lights are also possible triggers.

- Hormonal changes in women: Fluctuations in estrogen, such as before or during menstrual periods, pregnancy and menopause, seem to trigger headaches in many women.

- Sensory stimuli: Bright lights and sun glare can induce migraines, as can loud sounds. Strong smells-including perfume, paint thinner, secondhand smoke and others - trigger migraines in some people.

- Sleep changes: Missing sleep, getting too much sleep or jet lag can trigger migraines in some people.

- Physical factors: Intense physical exertion, including sexual activity, might provoke migraines. Tiredness and insufficient sleep, shoulder or neck tension, poor posture, and physical overexertion have all been linked to migraines. Low blood sugar can also act as triggers.

- Medications. Oral contraceptives and vasodilators, such as nitroglycerin, can aggravate migraines. Hormonal medications, such as oral contraceptives and hormone replacement therapy, also can worsen migraines.

- Food additives: These include the sweetener aspartame and the preservative monosodium glutamate (MSG), found in many foods. Food additives like nitrates (in pepperoni, hot dogs, and lunchmeats) may be responsible for up to $30 \%$ of migraines.

- Irregular mealtimes and dehydration have also been potential triggers.

True migraine headaches are not a result of a brain tumor or other serious medical problem. Only a health care provider who specializes in headaches can determine if your symptoms are due to a migraine or other condition.

\section{Are Migraine Headaches Hereditary?}

Yes, migraine headaches seem to run in families. Four out of 5 people with the condition have other family members who have them. If one parent has a history of these type of headaches, their child has a $50 \%$ chance of getting them, and if both parents have them, the risk jumps to $75 \%$.

\section{Types of migraine headcahe}

The terms for two types of migraine headaches refer to the symptoms that signal when one is about to start, called an aura.

- Migraine with aura (known as "classic" migraine)

- Migraine without aura (known as "common" migraine)

Migraine without aura: This type of migraine used to be called common migraine. Most people with migraine do not experience an aura. According to the International Headache Society, people who have migraine without aura have had at least five attacks that have these characteristics:

- Headache attack usually lasting 4 to 72 hours if it is not treated or if treatment does not work.

- Headache has at least two of these traits:

- it occurs only on one side of the head (unilateral)

- pain is pulsating or throbbing

- pain level is moderate or severe

- pain gets worse when you move, like when walking or climbing stairs

- Headache has at least one of these traits:

- it makes you sensitive to light (photophobia)

- it makes you sensitive to sound (phono phobia)

- you experience nausea with or without vomiting or diarrhea.

Migraine with aura: This type of migraine used to be called classic migraine, complicated migraine, and hemiplegic migraine. Migraine with aura occurs in 25 percent of people who have migraines. According to the International Headache Society, you must have at least two attacks that have these characteristics:

- An aura that goes away, is completely reversible, and includes at least one of these symptoms:

- visual problems (the most common aura symptom)

- sensory problems of the body, face, or tongue, such as numbness, tingling, or dizziness 
- speech or language problems

- $\quad$ problems moving or weakness, which may last up to 72 hours

- brainstem symptoms, which includes:

- difficulty talking or dysarthria (unclear speech)

- vertigo (a spinning feeling)

- tinnitus or ringing in the ears

- hypoacusis (problems hearing)

- diplopia (double vision)

- ataxia or an inability to control body movements

- decreased consciousness

- eye problems in only one eye, including flashes of light, blind spots, or temporary blindness (when these symptoms occur they're called retinal migraines)

- An aura that has at least two of these traits:

- at least one symptom spread gradually over five or more minutes

- each symptom of the aura lasts between five minutes and one hour (if you have three symptoms, they may last up to three hours)

- at least one symptom of the aura is only on one side of the head, including vision, speech, or language problems

- aura occurs with the headache or one hour before the headache begins

- Headache is not caused from another health problem and transient ischemic attack has been excluded as a cause.

- An aura usually occurs before the headache pain begins, but it can continue once the headache starts. Alternatively, an aura may start at the same time as the headache does. An aura can start 1 hour before the pain and usually last for 15 minutes to 1 hour. Visual auras include:

- Bright flashing dots or lights

- Blind spots

- Blurry vision

- Temporary vision loss

- Wavy or jagged lines

Other auras can affect your other senses. One might just have a "funny feeling" and not be able to describe the sensation, One could also have ringing in the ears or changes in smell (such as strange odors), taste, or touch.

Rare migraine conditions include these types of auras:

Hemiplegic migraine: A short period of paralysis (hemiplegia) or weakness on one side of the body. One might also feel temporary numbness, dizziness, or vision changes. If one gets these symptoms, it is important to know how to tell them apart from the signs of a stroke, which can seem similar.

Ophthalmic migraine: This is a rarer type of migraine with aura, but unlike other visual auras, it affects only one eye. Short-lived, partial, or complete loss of vision in one eye, along with a dull ache behind the eye, which may spread to the rest of your head. The International Headache Society defines retinal migraines as attacks of fully reversible and temporary vision problems in only one eye. These vision problems usually occur within an hour of the headache. Sometimes optical migraines are painless. Most people who have an optical migraine have had another type of migraine before. Other symptoms of this rare type of migraine include a droopy eyelid, double vision, or other vision changes.

Migraine with brainstem aura: Dizziness, confusion, or loss of balance can happen before the headache. The pain may affect the back of your head. These symptoms usually start suddenly and can happen with trouble speaking, ringing in the ears, and vomiting. This type of migraine is strongly linked to hormone changes and mainly affects young adult women.

Status migraineurs: This rare and severe type of migraine can last for more than 72 hours. The pain and nausea are so intense that one might need to go to the hospital. Sometimes medicines, or medication withdrawal, can cause them.

Acute migraine: Acute migraine is a general term for episodic migraines, that are not diagnosed as chronic. People who have episodic migraines have headaches up to 14 days a month. Thus, people with episodic migraines have fewer headaches, in a month than those with chronic ones.

Chronic migraine: This refers to any migraine that triggers attacks on over 15 days of the month. Chronic migraine used to be called combination or mixed headache because it can have features of migraine and tension headaches. It's also sometimes called severe migraine and can be caused by medication overuse. People who have chronic migraines have a severe tension or migraine headache more than 15 days a month for 3 or more months. More than eight of those headaches are migraines with or without aura. Compared to people who have acute migraines, people with chronic migraines are more likely to have: severe headaches, more disability at home and away from home, depression, another type of chronic pain, like arthritis, other serious health problems (comorbidities), such as high blood pressure, previous head or neck injuries etc....

Menstrual migraine: This is when the attacks occur in a pattern connected to the menstrual cycle. Menstrual-related migraines affect up to 60 percent of women who experience any type of migraine. They can occur with or without an aura. They can also occur before, during, or after menstruation and during ovulation. Research has shown that menstrual migraines tend to be more intense, last longer, and have more significant nausea than migraines not associated with the menstrual cycle. In addition to standard treatments for migraines, women with menstrual-related migraines may also benefit from medications that affect serotonin levels as well as hormonal treatments.

\section{Stages of Migraine}

Migraines often develop in distinct stages, although not everyone goes through all of these:

Prodromal (pre headache) stage: changes in mood from depression to euphoria, energy levels, behaviors like frequent yawning and appetite with certain type of food cravings, constipation, neck stiffness and increased thirst and urination that can occur several hours or days before an attack. 
Aura: When migraines with aura affect vision, the patient may see things that are not there, such as transparent strings of objects. They may also not see parts of the object in front of them or even feel as if part of their field of vision appears, disappears, and then comes back again. Usually visual problems, such as flashes of light or blind spots, which can last for 5 minutes to an hour. For some people, aura might occur before or during migraines. Auras are reversible symptoms of the nervous system. They're usually visual, but can also include other disturbances. Each symptom usually begins gradually, builds up over several minutes and lasts for 20 to 60 minutes. Examples of migraine aura include: Visual phenomena, such as seeing various shapes, bright spots or flashes of light; Vision loss; Pins and needles sensations in an arm or leg; Weakness or numbness in the face or one side of the body; Difficulty speaking; Hearing noises or music; Uncontrollable jerking or other movements.

Headache stage: usually a pulsating or throbbing pain on 1 side of the head, often accompanied by feeling sick, nausea and vomiting or extreme sensitivity to bright light and loud sounds, which can last for 4 to 72 hours A migraine usually lasts from four to 72 hours if untreated. How often migraines occur varies from person to person. Migraines might occur rarely or strike several times a month.

Resolution stage: when the headache and other symptoms gradually fade away, one might feel tired for a few days afterwards. Some people report feeling elated. Sudden head movement might bring on the pain again briefly.

\section{Symptoms of Migraine Headache}

- A headache that often begins as a dull ache and grows into throbbing pain. It usually gets worse during physical activity. The pain can shift from one side of the head to the other, can be in the front of the head, or feel like it's affecting one's entire head.

- Sensitivity to light, noise, and smells

- Nausea and vomiting, upset stomach, and belly pain

- Loss of appetite

- Feeling very warm or cold

- Pale skin

- Fatigue
- Dizziness and Alteration of consciousness

- Blurred vision

- Diarrhea

- Fever (this is rare)

- Confusion state

- Light-headedness

- Scalp tenderness

- Syncope

- Seizure

- Photopsia

- Fortification spectra

- Paresthesia

- Vertigo

Most migraine headaches last about 4 hours, but severe ones can go for more than 3 days. How often they happen differs for everyone, but it's common to get two to four headaches per month. Some people may get migraine headaches every few days, while others get them once or twice a year.

\section{Diagnosis}

To help identify a migraine headache, it can be useful to keep a diary of symptoms noting the time of onset, any triggers, the duration of the headaches, any noticeable signs or auras leading up to a migraine attack, and any other symptoms. A migraine can cause severe throbbing pain or a pulsing sensation, usually on one side of the head. It is often accompanied by nausea, vomiting, and extreme sensitivity to light and sound. Migraine attacks can last for hours to days, and the pain can be so severe that it interferes with your daily activities. If one has migraines or a family history of migraines, a doctor trained in treating headaches (neurologist) will likely diagnose migraines based on the medical history, symptoms, and a physical and neurological examination. The International Headache Society recommends the " $5,4,3,2,1$ " criteria to diagnose migraines without aura. This stands for:

- 5 or more attacks with a duration of 4 hours to 3 days

- At least two of the following qualities: Occurring on one side of the head, a pulsating quality, moderate-tosevere pain, and aggravation by routine physical activity

- At least one additional symptom, such as nausea, vomiting, sensitivity to light, or sensitivity to sound.

Table 15-4: Simplified Diagnostic Criteria for Migraine

\begin{tabular}{|c|c|}
\hline \multicolumn{2}{|l|}{$\begin{array}{c}\text { Repeated attacks of headache lasting 4-72 } \mathrm{h} \text { in patients with a normal physical examination, no other reasonable cause for the headache, } \\
\text { and }\end{array}$} \\
\hline At least 2 of the following features & Plus at least 1 of the following features \\
\hline Unilateral pain & Nausea/vomiting \\
\hline Throbbing pain & Photophobia and phono phobia \\
\hline Aggravation by movement & \\
\hline Moderate or severe intensity & \\
\hline
\end{tabular}

\section{Investigations}

If the condition is unusual, complex or suddenly becomes severe, tests to rule out other causes for pain might include:

- MRI: An MRI scan uses a powerful magnetic field and radio waves to produce detailed images of the brain and blood vessels. MRI scans help to diagnose tumors, strokes, bleeding in the brain, infections, and other brain and neurological conditions.

- CT scan: A CT scan uses a series of X-rays to create detailed cross-sectional images of the brain. This helps to diagnose tumors, infections, brain damage, bleeding in the brain and other possible medical problems that may be causing headaches.

\section{Management}

When symptoms of migraine start, try heading to a quiet, darkened room. Close your eyes and rest or take a nap. Place a cool cloth or ice pack wrapped in a towel or cloth on your forehead or at the back of your neck. Other practices that might soothe migraine with aura pain include: 
- Develop a sleeping and eating routine: Don't sleep too much or too little. Set and follow a consistent sleep and wake schedule daily. Try to eat meals at the same time every day.

- Drink plenty of fluids: Staying hydrated, particularly with water, might help.

- Keep a headache diary: Continue recording in your headache diary even after you see your doctor. It will help you learn more about what triggers your migraines and what treatment is most effective.

- Exercise regularly: Regular aerobic exercise such as walking, swimming and cycling reduces tension and can help prevent a migraine. Warm up slowly, however, because sudden, intense exercise can cause headaches. Regular exercise can also help you lose weight or maintain a healthy body weight, and obesity is thought to be a factor in migraines.

\section{Homoeopathic Therapeutics}

Homoeopathy depends upon totality of symptoms but there are some important medicne for migraine disscued below-

Aconitum Napellus: Burning in the forehead as if everything could be pushed out; pulsation in the left side of the forehead, as if from severe blows on the right side of the forehead. ;<motion, drinking, talking, sunlight;>lying with head high.

Aranea Diadema: Headache at regular intervals, with flickering before the eyes, vertigo and a feeling as if the hands and head were bloated and swollen; coldness of hands and feet;> open air.

Argentum Nitricum: Boring pain in the head, <left frontal eminence,> by tight bandaging; caused by mental emotions; depression of nervous force; bones of head feel as if they were separating, or head feels as if it were enormously large, frequently ending in vomiting or sour fluid.

Arsenicum Album: Throbbing, stupefying headache over the left eye, of such severity as to render him angry and irritable, temporarily >by cold water on head,<from wrapping up head warm, intermitting, tearing, boring, burning pain in right supraorbital region, extending over the eye and into the upper teeth,> by walking about; periodical pain above left eyebrow and temple, followed by vomiting of a yellow, bitter or tenacious mass.

Belladonna: Violent hyperemia, with throbbing carotids, red face, intolerance of least jar, noise or light; hemiopia, retinal blindness, slight paralysis of tongue, even transitory hemiplegia. Attacks more in the afternoon or evening; before or during menses, with dysmenorrhea, globus hystericus, titillating cough; all> in bed in a dark room, far removed from light and noise.

Bromium: Hemicrania, affecting left side only, of a heavy pressing character, < from stooping, in the sun and after drinking milk;> in shade and by pressing the neck.

Calcarea Carbonica: Nausea, eructation's, icy coldness in head; pains begin in the morning, often of the side on which the patient rested, pressing pain from within outward, as if head would split, tearing headache above eyes down to the nose, with nausea; hemicrania, left side, stitches in temple and downward into the teeth, $<$ on awaking, from motion, noise and talking,>in evening, especially in chlorotic girls, with much empty eructation's and inclination to vomit; headache from occiput to vertex, with fullness and heaviness in head; < from any stimulant, as liquors or excess in venery, $>$ by rest, quiet and darkness.

Capsicum Annum: Flushes at the start, then face pale and sunken; stitching, tearing, drawing pains with sensation as if the skull would burst; at height of attack, nausea, vomiting, anguish, < from motion, stooping, light, >in fresh air, from cold; patient feels lazy and shuns all motions, wants to remain indoors.

Causticum: Hemicrania with nausea and vomiting; pain mornings when awaking, by motion of head and rapid walking, increasing progressively and suddenly diminishing; constant succession of shocks and jerks in head; rheumatic pain in head causes nausea.

Cocculus Canadensis: Migraine with vertigo and nausea, pain especially in frontal protuberance and left orbit, <eating, drinking, starting up, walking in fresh air, sickheadache from riding in a carriage, boat, trains or cars; headache at each menstrual period, with nausea and inclination to vomit.

Colocynthis: Hemicrania from anger with indignation, after suppression of menses, pains extending to the forehead and left side of the head, with nausea, vomiting and diarrhea, especially towards the evening; violent, periodical or intermittent headache; bilious, gouty or nervous migraine of great severity.

Conium Maculatum: Sick-headaches with inability to urinate; great giddiness, < lying on the bed, when everything in room seems to go round, numbness, with sensation of coldness, on one side of the head.

Gelsemium Semperviens: Hemicrania with dim-sight and double vision, or preceded by great sensitiveness to noise, coming on suddenly with vertigo and great heaviness of head, semi-stupor, settled, dull heavy pain in the occiput, mastoid process and upper cervical region, down to the shoulders, > when sitting by reclining head high on the pillow, < by exertion or study; paroxysms end with profuse urination.

Glonoinum: Hemicrania from excessive use of wine; nausea, is obliged to sit down, dimness before eyes, like a cloud, followed by most violent headache>vomiting.

Ipecacuanha: Sick-headache; brain feels bruised and as if it were drawn down to the root of the tongue and into teeth; nausea and vomiting, > outdoors.

IRIS Versicolor: Attacks begin with blurring of sight or speck before the eye of the affected side, a stupid stunning headache, neuralgia of infraorbital or dental nerves, attended with sour, watery vomiting, from mental exhaustion; < towards evening from cold air; > from moderate motion. 
Kali Bichromicum: Blindness followed by violent headache, must lie down; aversion to light and noise, sight returns with the increasing headache; frontal headache over one eye, shooting at intervals in right temple; semi-frontal headaches in small spots; nausea with a feeling of heat over the body and bitter, sour, glairy vomiting.

LAC Caninum: Pain especially over the left eye, < noise and talking, >rest and cold water; pain as from knife thrust over the left zygoma upto the vertex; neuralgic pain in the left side of the head, followed by a film over the right eye, with inclination to rub it off; intense darting pain around the left eye; empty weak feeling in the pit of the stomach.

Melliolotus ALBA: Intense frontal headache, preceded by hot flushed face, < forenoon, with some fever, periodically recurring every few days, $>$ by epistaxis or menstrual flow.

Mercurius: Migraine from cold; drawing, tearing pains in the head, stitches in the ears and teeth, fullness and heaviness in the head, chilliness with cold hands and hot cheeks, without heat in head and without thirst; sweat does not relieve, < in bed, by noise or touch,> by rest and external application of heat.

Natrium Muriaticum: Attacks begin in the morning in the bed, diminishes after rising and moderate exercise, but becomes worse from rapid motion, accompanied by nausea and vomiting of transparent phlegm or water; dull heavy headache with protrusion of tears and drowsiness; sleep not refreshing; headache before, during and after menses; pain as if head would burst $<$ coughing, loss of consciousness and twitching of limbs.

Natrum Sulphuricum: Sick-headache with bilious diarrhea and vomiting of bile; bitter taste in the mouth, congestion of blood to head, with fullness and heat in the vertex, as if it would split; vomiting, <motion and reading, >quiet. Menstrual migraine; attacks recurring every spring and sudden in their onset; boring pain in the right temple. Preceded by burning in the stomach, bitter taste, languor.

Nux Vomica: Gouty and hemorrhoidal patients. Attack sets in the morning on awakening, getting worse during the day; nausea and vomiting during the attack; becoming often most severe in the occiput; drawing, aching feeling as of a nail driven into the head, or as if the brain were dashed to pieces; face pale, or sallow on a red ground; <from mental exercise, by motion and by rest, from stimulants; patient irritable with his abdominal plethora.

Oleum Animale Migraine with polyuria, urine perfectly clear, face suffused, red; eyes heavy and bloodshot, can hardly lift the upper eyelids; speech thick and unwieldy; patient makes impression of under influence of liquor; pulse full and round.

Phosphorus: Headache over the left eye, with black spots which dance before eyes; transient blindness; headache with vomiting, unilateral, pressing, pulsating, boring pain in one or other temple, radiating into forehead, eyes and vertex, with sensation of painful nodes on scalp $<$ morning and evening, with yawning, turgor of face and watery urine, from talking, in warm room or after eating,> in fresh air, when resting or sleeping; exhausting after attack and tendency to catch cold.

Pulsatilla: General chilliness; scanty menses; bursting, throbbing or boring, stitching pains on one side of the head, obscuration of sight, white tongue, nausea and vomiting, thickly furred tongue with clammy mouth and relief from cold air; shifting pains < evenings.

Rhus Toxicodendron: Catching cold from dampness; tearing, stitching pain in one temple radiating to ear, cheek and teeth as well as towards occiput, with heat, fullness and heaviness of the head and sensation of looseness of the brain $<$ after midnight and after eating, by moving head, cold air or emotions,> by rest in bed and warmth.

Sanguinaria Canadensis: Migraine in women who menstruate freely; excessive pains with bilious vomiting, electric stitches through head, toothache, earache, pains in extremities, chills, preceded by scanty and followed by copious clear urination, attacks begin in the morning and gets worse during the day, reaching their acme at the noon, pains are more right-sided; attack every seventh day, the veins of the suffering temple full of blood and sensitive to touch.

Spigelia Antihelminthica: Terrible pain in globe of the eye as if it were torn out; dilatation and immobility of the pupil; temporary blindness, boring, tearing, stitching pains in the forehead, temples, vertex or occiput, especially left side, following the course of the trifacial nerve and producing there paretic sensations; dull stitches from within outwards on top of the head < from touch, or after washing, but > while washing.

Stannum Metallicum: Neuralgic headache every morning over one or the other eye, mostly left, gradually spreading over whole forehead, gradually increasing and decreasing; painful jerks through left temple, forehead and cerebellum, leaving a dull pressure, < during rest, > vomiting of bile and mucus mornings and from motion.

Theridion Curassavicum: Flickering before the eyes; nausea< by closing the eyes and by noise; extreme hyperesthesia of acoustic nerve, throbbing over the left eye and across forehead, sick stomach, < on rising from lying; desire for stimulants.

Veratrum Album: Neuralgia of head with indigestion; features sunken, fainting with cold sweats and great thirst; pains drive to despair.

\section{Repertorial hints for rubrics on migraine}

PAIN, hysterical headache: Asaf., aur., bell., bry., cimic., cocc., coff., gels., hell., hep., hyos., ign., kali bi., lach., lact., mag-c., $\operatorname{mag}-\mathrm{m}$., mosch., nux-m., nux-v., ph-ac., phos., plat., menses, before: Acon., agn., alum., amc., asar., bell., bor., bov., brom., bry., bufo., calc-p., calcs., calc., carb-an., carb-v., caust., cimic., cinnb., cupr., ferrar., ferr-i., ferr., gels., glon., graph., hep., hyper., iod., kali- 
p., Kreos., lac-c., lac-d., lach., laur., lil-t., lyc.,nat-c., natm., nit-ac., nux-m., plat., puls., sep., sulph., thuj., Mental exertion, from : Acon., agar., $\arg -\mathrm{m} ., \arg -n$. , arn., ars i.,asar., Aur., bell., bry., Calc-p.,Calc., carb-v., chin., cimic., cocc., coff., dig., gels., Glon., ign., kali-p., lac-c., lach., Lyc., $\operatorname{mag}-c$., mag-m., Nat-c., Nat-m., Nat-p., nats., Nux-v., Ph-ac., phos., Pic-ac., psor., Puls., sabad., sep., Sil., spig., staph., stram., sulph.,., ther., noise, from : Acon., agar., anac., arn., ars., Bell., bor., bry., cact., Calc., chin., cocc., coff., con., ferr-p., gels., kali bi., kali-p., kalis., lac-c., lac-d., lach., Nat-a., nat-c., Nit-ac., nux-v.,phos., sang., sil., spig., stann., tab., Ther., zinc.

odors, from strong: Acon., anac., aur., bell., cham., chin., coff., colch., graph., ign., lyc., nux -v., phos., sel., sil., sulph. periodic headache: Act-sp., aeth., aloe., Alum., ambr., ammc., anac., apis., aran., arn., ars-i., Ars., asaf., bell., benz-ac., cact., calc-s., calc., carb-v., Cedr., cham., china.,Chin., Coloc., cupr., eup-per.,ferr-ar., ferr., ign., kaliar., kali bi., kreos., lac-d., lach., laur., l lob., lyc., nat-a., Nat-m., Nit-ac., phos., puls., rhust., Sang., Sep., Sil., spig., stram., sulph., tab., tub., zinc.

every day: Ars., bell., calc., lyc., nat-m., nux-m., nux-v., phos., sil., spig., stann., sulph..

pulsating: Acon., Bell., calc-p., Carb-s., Carb-v., Chins., Chin., Ferr., gels., Glon., kali-c., kali-p., lach. led., Lyc., lyss., Nat-m., nux-v., op., Psor., Puls., Sulph., thunderstorms, before: Bry., lach., nat $c$., Phos., rhod., sep., sil.

weather, from changes of: Ars., bry., calc $-p$., calc., carb-v., lach., mez., nux-m., ph-ac., phos., psor., ran-b., rhod., Rhus-t., sil., verb., vip.

PAIN, forehead, right side: Acet-ac., acon., agar., aloe., anac., ant -t., apis., arg -n., arn., ars., bell., chel., glon., hep., nicc., ol-an., op., osm., phyt., pic-ac., Prun-s., psor.,ruta.,sang.,sep., sil., stram., sul-ac., sulph.,thuj., zinc. left side : asar., bell., glon., lac $-c$., lyc., nat-c., nat-m., psor., rhus t., sep., stann., staph., sul-ac., sulph., Thuj.,.

Boring, digging, screwing: arg-n., bell., bry., Caust., ., coloc., hep.,kali-p., nat-m., nat-s., nit-ac. puls., rhust.,sep., sil., spig., sulph., tab., thuj.

Bursting: Bell., Bry., Calc., caust., Chin., coff., Con.., euphr. Glon., graph., Lach., lyc., Lyss.; Merc., Nat-m., nicc., Phos., puls. Sep., sulph.,

Drawing: agar. ars., bry., calc-p., calc., Chin., gels., graph., Merc., nat-m., Nux-v., phos., plat., plb., puls., rhod., rhus -t., sep., sil., Sulph.

Jerking: arn., Bell, bry., carb-ac., nat-m., puls., sep.,spig., Sulph.,

Lancinating: ars., bell.cupr.,

Pressing: agar., ant t., arg-n., arn., Bell., bism., bry., calc., camph., caps., carb-ac., carb-an., Carb-v., caust., Chin.,., cocc., coff., coloc., con., Glon., hell., kali-c.kali-p.,Lac-c., Lach., lyc., Merc., Nat-m., nat-s., nicc., Nit-ac., Nux-v., olnd.,,Psor., Puls., rhod., rhus-t., ruta., sabad., sep., sil., spig., sul-ac., Sulph., tab., ther., thuj.

Shooting (See Cutting, Lancinating, Shocks, Stitches, etc.) alum., bell., carb-v., con., eup-per., ferr., gels., hell., kalibi., Kali-c., nat-c., nat-m., rhus-t., sep.,

Stitching: Acon., agar., agn., aloe. arn., ars., bell., bry., calc., caust., Chin., cic., cocc., con., cupr., cycl., dig. dulc., elaps., eug., euon., euphr., ferr-p., ferr., gels., glon., ign., kali-bi., Kali-c.., Kali-p., Kali-s., lach., lachn.,, lyc., magc., mag-m., mang. merc., nat-c., nat-m., nat-p., nat-s., nicc., nux-v.,, ph-ac., phos., Puls., rhus-t.,, sabin., sars., sep., sil., spig., squil., stront., sul-ac., Sulph., thuj., valer.

\section{Conclusion}

It is estimated that $5 \%$ population suffers from migraine. In most people it recurs again and again as frequently which disrupt the normal day to day activity of people and produce anxiety and other sufferings. As we know that it will be provocated by different exciting factors and hidden maintaining cause. So, we have to remove that cause and by doing so the effect will be remove automatically as cited in Organon of Medicine. Homoeopathy play the important role for these types of disease. Large number of medicine which was verified frequently in healthy prover by which we can rely on that drug and it also clinically verified. The selection of medicine is strictly on the basic principle SIMILIA SIMILIBUS CURENTUR, the efficacy of the drug helps to gain confident as well as helps in future study. By the only medicine its not possible to control any disease but we must follow proper diet and regimen as well as mental exertion

\section{References}

1. Harrison's. Principles of Internal Medicine, 17th edition, Mc Graw Hill Medical 2008;1:96-98.

2. Davidson's. Principles and practice of Medicine, 19th edition; New York: Churchill Livingstone. 2002, 1118,1119 .

3. Whitmarsh TE, Coleston-Shields DM, Steiner TJ. Double-blind randomized placebo-controlled study of homoeopathic prophylaxis of migraine. Pub Med 1997;17(5):600-4.

4. Brigo B, Serpelloni G. Homeopathic treatment of migraine: A randomized double-blind controlled study of sixty cases (homeopathic remedy versus placebo). Berlin Journal in Research of Hornoeopathy 1991;1:98- 
106.

5. Boericke William. Pocket Manual of Homoeopathic Materia Medica \& Repertory, New Delhi: B. Jain Publishers (P) Ltd, 2016, 702, 578, 650, 196, 355, 537, 267, 484, 535, 481, 532.428, 671, 404.305, 79, 185.

6. Allen HC, Keynotes Rearranged and Classified with Leading Remedies of the Materia Medica and Bowel Nosodes, 10th edition, New Delhi: B. Jain Publishers (P) Ltd., 2013. P. 119, 213, 285, 195, 239, 56, 38, 283, 104, 310, 135, 159.

7. Clarke JH. Dictionary of practical materia medica; New Delhi: B Jain Publishers (P) Ltd; Reprinted edition. 1998.

8. Kent JT, Repertory of the Homoeopathic Materia Medica. Kolkata: Medical Book Publishers 2017, 140, 150, 271, 508.

9. Schroyens Frederik. Synthesis - Repertorium Homoeopathicum Syntheticum; B Jain Publishers (P) Ltd., New Delhi; Edition 9.1. 